\title{
Expression of FGFRL1, a novel fibroblast growth factor receptor, during embryonic development
}

\author{
BEAT TRUEB and SARA TAESCHLER
}

\author{
ITI Research Institute, University of Bern, Murtenstrasse 35, CH-3010 Bern, Switzerland
}

Received October 7, 2005; Accepted December 12, 2005

\begin{abstract}
FGFRL1 is a novel member of the fibroblast growth factor receptor (FGFR) family. To investigate its expression during mammalian embryonic development, we have used the mouse system. Expression of Fgfrl1 is very low in mouse embryos of day 6 but steadily increases until birth. As demonstrated by in situ hybridization of 16-day-old embryos, the Fgfrl1 mRNA occurs in cartilaginous structures such as the primordia of bones and the permanent cartilage of the trachea, the ribs and the nose. In addition, some muscle types, including the muscles of the tongue and the diaphragm, express Fgfrl1 at relatively high level. In contrast, the heart and the skeletal muscles of the limbs, as well as many other organs (brain, lung, liver, kidney, gut) express Fgfrl1 only at basal level. It is conceivable that Fgfrl1 interacts with other Fgfrs, which are expressed in cartilage and muscle, to modulate FGF signaling.
\end{abstract}

\section{Introduction}

FGFRL1 is a recently discovered member of the fibroblast growth factor receptor (FGFR) family (1). This family of transmembrane receptors is known to control the proliferation, differentiation and migration of cells in various tissues including bone, cartilage and muscle. The gene for FGFRL1 is found in all vertebrates from fish to man, but it appears to be missing in invertebrates (2).

Originally, we isolated cDNA clones for FGFRL1 from a subtracted cDNA library that had been prepared with the aim to identify novel cartilage-specific genes (1). Independently, two other research groups identified clones for the same receptor in cDNA libraries prepared from human embryos (3) and murine lymph node cells (4) and termed the novel protein FGFR5. The structure of FGFRL1/FGFR5 is highly related to that of the other four FGFRs $(5,6)$. It contains a signal peptide, three extracellular Ig-like domains and a single transmembrane domain. In contrast to the FGFRs, however,

Correspondence to: Dr Beat Trueb, ITI Research Institute, University of Bern, Murtenstrasse 35, P.O. Box 54, CH-3010 Bern, Switzerland

E-mail: beat.trueb@iti.unibe.ch

Key words: cartilage, FGF, FGFR, fibroblast growth factor receptor, in situ hybridization the novel receptor is missing the intracellular tyrosine kinase domain, which would be required for signal transduction by transphosphorylation (5-8). Instead it contains a short, histidine-rich domain at its C-terminus that is not related to any other protein. Owing to the structural relationship of the extracellular domain and to the lack of the kinase domain, we have speculated that FGFRL1 might function as a decoy receptor and modulate FGF signaling. Recombinant FGFRL1 is able to interact with FGF2 $(3,7)$, an observation that is in line with our assumption. Moreover, FGFRL1 has a negative effect on cell proliferation when overexpressed in MG63 osteosarcoma cells, lending further support to the idea of a decoy receptor. On the other hand, the affinity of FGF2 for the novel receptor is relatively weak $\left(\mathrm{K}_{\mathrm{d}} 10^{-8}\right)$, provoking questions about the nature of the authentic ligand under physiological conditions (7).

To learn more about the putative function of FGFRL1, we set out to investigate its expression during development. Since potent antibodies against the FGFRL1 protein are not yet available, we employed our cDNA clones for Northern blotting and in situ hybridization experiments to demonstrate the expression pattern of FGFRL1 during mouse embryonic development.

\section{Materials and methods}

Northern blots. Total RNA was isolated from various mouse tissues with the aid of the RNeasy kit from Qiagen (Hilden, Germany). The tissues were homogenized in guanidinium isothiocyanate buffer, extracted with phenol/chloroform, followed by chloroform (9) and loaded onto the RNeasy columns. Purified RNA (10 $\mu \mathrm{g} / \mathrm{lane})$ was separated on $1 \%$ agarose gels in the presence of formaldehyde and transferred to Nylon membranes by vacuum blotting. The membranes were hybridized at $42^{\circ} \mathrm{C}$ with the labeled cDNA probe in a buffer containing $50 \%$ formamide (10). After $24 \mathrm{~h}$, the blots were washed and exposed to X-ray film or analyzed with a phosphorimager (Storm 840, Molecular Dynamics, Sunnyvale, CA). The probe corresponded to an $\mathrm{XbaI} / \mathrm{BamHI}$ fragment of the mouse Fgfrl1 cDNA (position 661-1417) that had been labeled with $\left[\alpha^{-32} \mathrm{P}\right]$ by the random primed oligolabeling method (11).

In situ hybridization. In situ hybridization experiments were performed essentially as described by Wälchli et al (12) with labeled RNA probes. Samples from 15- to 17-day-old mouse embryos were embedded in paraffin and cut into serial sections. 


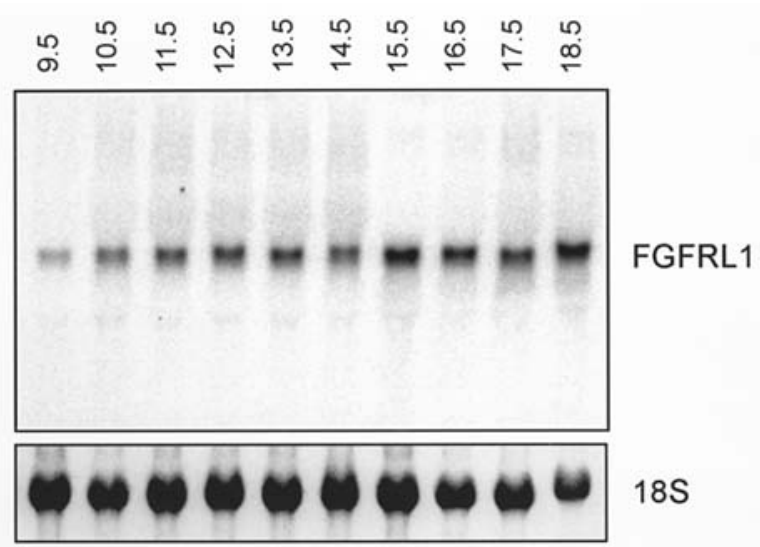

Figure 1. Expression of Fgfrl1 during mouse embryonic development. Total RNA from mouse embryos of 9.5-18.5 days post coitum was resolved on an agarose gel and transferred to a Nylon membrane. The membrane was hybridized with a radioactively labeled cDNA probe for mouse Fgfrl1. The panel at the bottom shows the $18 \mathrm{~S}$ ribosomal RNA stained with ethidium bromide as a loading control.

The mouse Fgfrl1 cDNA sequence (see above) was placed into the $\mathrm{pSK}^{+}$vector and riboprobes were transcribed by $\mathrm{T} 7$ (anti-sense) or T3 (sense) RNA polymerase in the presence of ${ }^{35}$ S-uridine 5 '-triphosphate. The tissue sections were digested with proteinase $\mathrm{K}$ and hybridized with the labeled probes at $60^{\circ} \mathrm{C}$. After $18 \mathrm{~h}$, the slides were treated with RNase A and washed with $0.1 \mathrm{X}$ standard saline citrate at $65^{\circ} \mathrm{C}$. The slides were then coated with NTB-2 emulsion (Eastman Kodak, Rochester, NY) and exposed for 3 days at $4^{\circ} \mathrm{C}$. After developing with D-19 developer, the sections were stained with hematoxylin and eosin. Finally, the slides were inspected under a Nikon Eclipse E1000 microscope equipped with dark field optics.

\section{Results}

Expression of Fgfrll during embryogenesis. To investigate the relative expression of Fgfrl1 during embryogenesis, a Northern blot containing total RNA from mouse embryos of days 9.5-18.5 was hybridized with a ${ }^{32} \mathrm{P}$-labeled cDNA probe (Fig. 1). Very low expression of Fgfrl1 was observed at day 9.5 as indicated by the faint band migrating with a relative mobility of 2600 nucleotides. This signal steadily increased until day 18.5. Prominent expression of Fgrl1 was observed between days 15.5 and 18.5.

Expression of Fgfrll in different tissues. Mouse embryos of different stages were cut into sagittal sections and hybridized with ${ }^{35}$ S-labeled RNA probes for mouse Fgfrl1 (Fig. 2). Control sections treated with the sense probe were consistently found to be negative (Fig. 2D). Sections from a 12.5-day-old embryo treated with the anti-sense probe did not reveal any distinct signal stronger than background. Sections from a 15.5-day-old embryo yielded a weak signal, which required electronic enhancement for clear visualization. Sections from 16.5- and 17.5-day-old embryos exhibited a prominent signal that could readily be photographed (Fig. 2A-C). Nevertheless, the signal was considerably weaker than the signal obtained
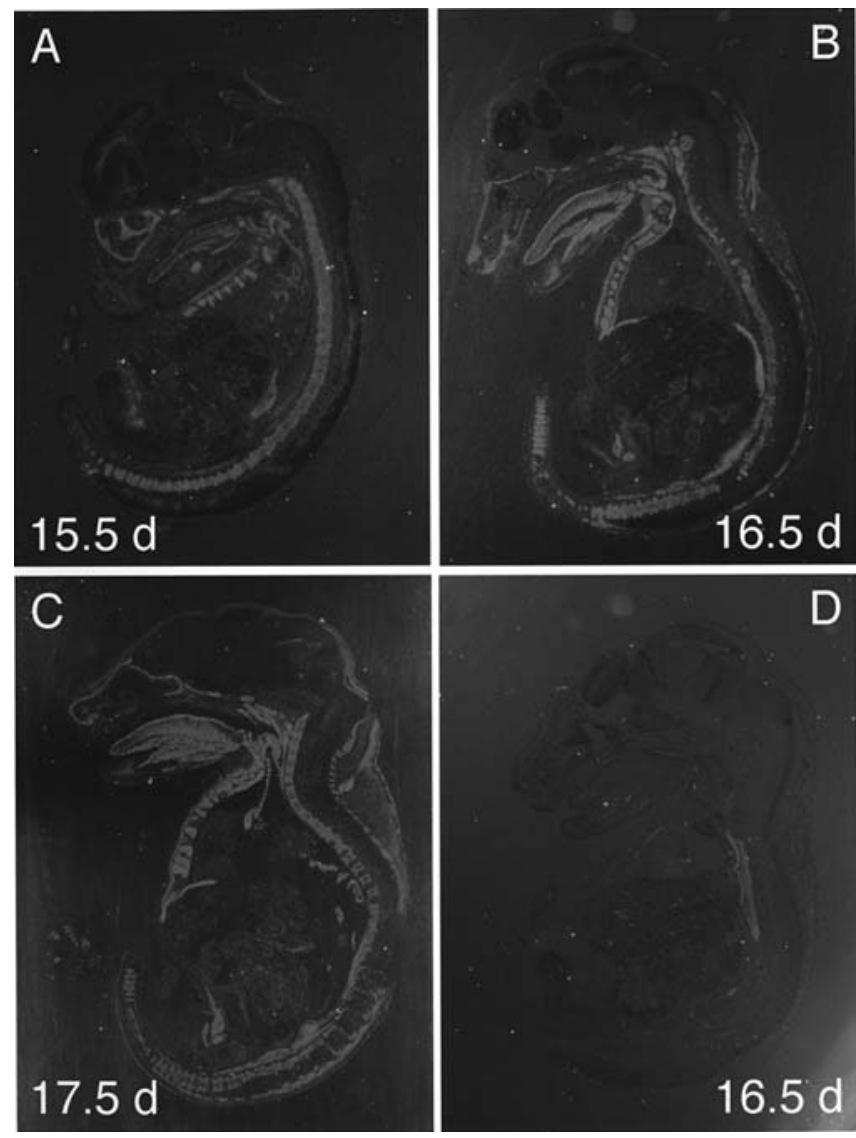

Figure 2. Expression of Fgfrl1 in late mouse embryos. Whole body sections of mouse embryos prepared at 15.5 (A), 16.5 (B and D) and 17.5 (C) days post coitum (16) were hybridized with radioactively labeled anti-sense (A-C) or sense (D) cRNA probes for mouse Fgfrl1. The hybridization signal was visualized under the microscope by dark field optics.

with a control probe for $\beta$-actin (data not shown). Hybridization with our Fgfrl1 probe was found to occur primarily in structures of bone and cartilage, including the primordia of the vertebral bodies, the primordia of the pelvic bone as well as the permanent cartilage of the nose, the ribs and the trachea. A distinct signal was also noted in some muscular structures, such as the muscles of the tongue and the diaphragm. In contrast, the brain, the spinal cord, the thymus, the lung, the heart, the liver and the gut showed only background signal (Fig. 2).

Selected structures were inspected at higher magnification (Fig. 3). In the paw of the hind limb (Fig. 3A and B), faint expression of Fgfrll was observed in the primordia of the metatarsal bones, while the skin, the tendons and the loose connective tissue in between were negative. In the trachea (Fig. 3C and D), all tracheal rings were positive. The silver grains appeared to be evenly distributed throughout the cricoid cartilage, whereas the perichondrium contained barely any signal. Likewise, the costal cartilage of the ribs had silver grains distributed over the entire area, whereas the perichondrium appeared to be negative (Fig. 3E and F). The muscles on top of the ribs (musculus pectoralis superficialis and musculus pectoralis profundus) as well as the intercostal muscles were also clearly positive. Furthermore, a faint signal was observed at the location of the pleura, but this signal is 

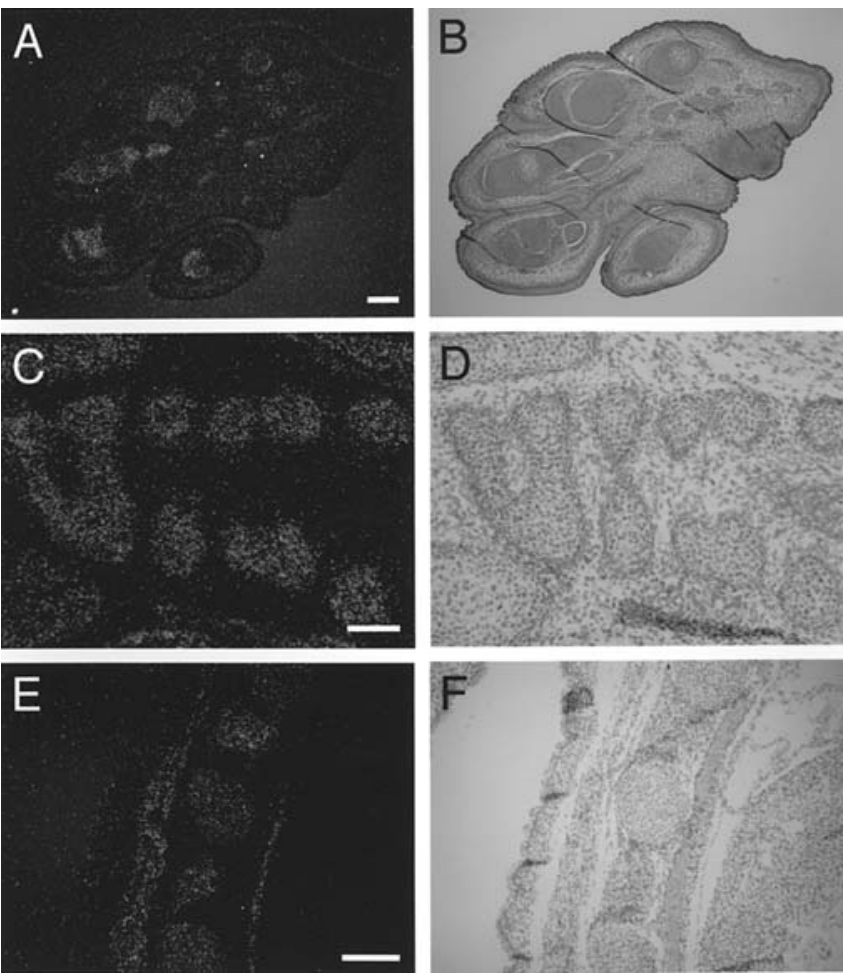

Figure 3. Expression of Fgfrl1 in cartilaginous structures. Selected sections from 17.5-day-old (A-D) and 16.5-day-old mouse embryos (E and F) were hybridized with an anti-sense probe for Fgfrl1 and inspected under dark field (A, C and E) and bright field (B, D and F) optics. (A) and (B) show consecutive, transversal sections through the distal part of the paw from the hind leg, scale bar $200 \mu \mathrm{m}$. (C) and (D) show a sagittal section through the chest depicting the tracheal rings (cricoid cartilage). Cranial is to the left, scale bar $100 \mu \mathrm{m}$. (E) and (F) represent a sagittal section through the chest depicting the ribs with costal cartilages 3,4 and 5 as well as intercostal muscles. Cranial is to the top, scale bar $200 \mu \mathrm{m}$.

difficult to interpret since it was also detected in the control section (Fig. 2D), but not in the section prepared from an older embryo (Fig. 2C).

The heart with ventricle and atrium appeared to be negative, but the aorta located just beneath the tracheal rings was prominently stained (Fig. 2C). At higher magnification, the silver grains appeared to be distributed evenly throughout the aortic wall (Fig. 4A and B). Likewise, the diaphragm contained silver grains distributed over the entire thickness of the muscle and did not reveal any substructures (Fig. 4C and D). An interesting pattern was observed with the tongue (Fig. 4E and F). While the tunica mucosa was negative and formed a black belt around the tongue, the longitudinal muscle bundles beneath the mucous membrane as well as the vertical muscle bundles spanning the tongue (13) were strongly positive and yielded a striking, striped pattern.

Northern blots. The results obtained by in situ hybridization were verified by Northern blotting experiments utilizing a ${ }^{32} \mathrm{P}$-labeled cDNA probe that corresponded to the probe used for in situ hybridization. Total RNA was extracted from various tissues of newborn mice (9-27 days) and resolved on two agarose gels. After blotting and hybridization, our probe was found to bind specifically to the 2600 nucleotide band as noted above (Fig. 5). A fairly strong signal was observed with RNA from the cartilaginous structures of the sternum and the
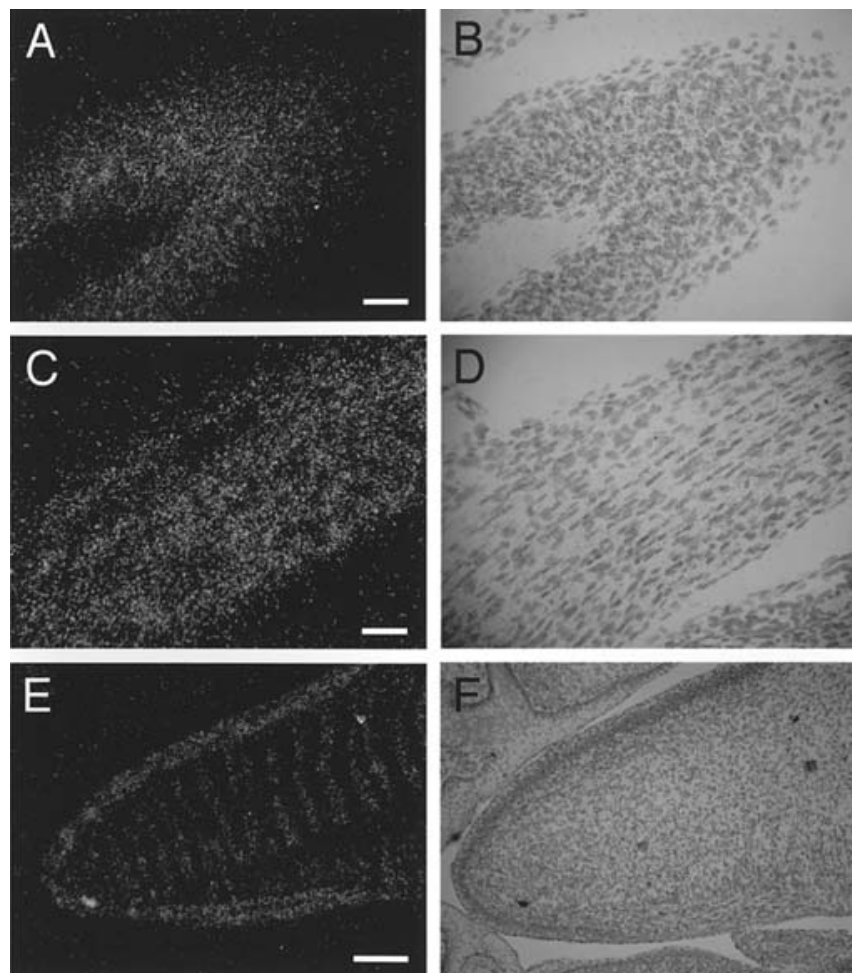

Figure 4. Expression of Fgfrl1 in muscular tissues. Selected sections from 17.5-day-old mouse embryos were hybridized with an anti-sense probe for Fgfrl1 and inspected under dark field (A, C and E) and bright field optics (B, D and F). (A) and (B) show a section through the aorta, scale bar $50 \mu \mathrm{m}$. (C) and (D) show a section through the diaphragm, scale bar $50 \mu \mathrm{m}$. (E) and (F) show a sagittal section through the tongue, scale bar $200 \mu \mathrm{m}$.
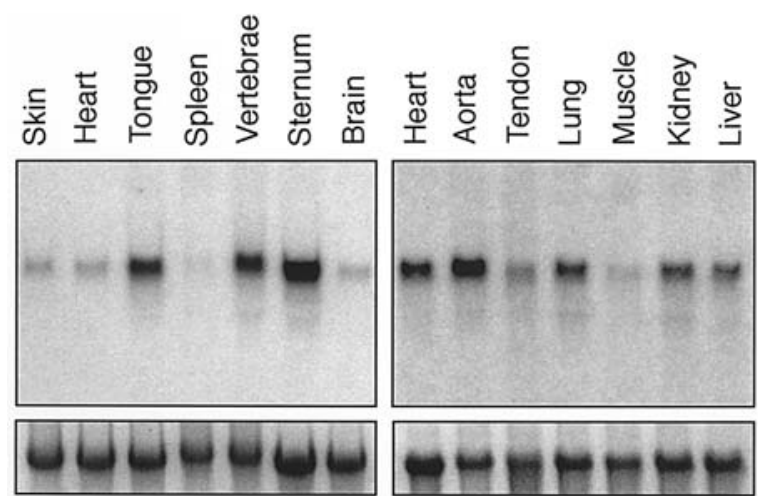

Fgfrl1

Figure 5. Expression of Fgfrl1 in several mouse tissues. Total RNA was extracted from new-born mice, separated on two agarose gels and transferred to Nylon membranes. The blots were hybridized with a cDNA probe for mouse Fgfrl1. Note that the blot on the right was exposed five times as long as the blot on the left to visualize faint expression. RNA from heart is shown on both blots and may serve as an internal reference. At the bottom, the $28 \mathrm{~S}$ ribosomal RNA stained with ethidium bromide is included as a loading control.

vertebral bodies. A prominent signal was also obtained with RNA from the tongue and the aorta (Fig. 5, left). Other tissues including skin, heart, brain, lung, kidney and liver revealed only a faint signal. In the cases of tendon, skeletal muscle (derived from the hind limb) and spleen, this signal was barely detectable after normal exposure time, but became visible after prolonged exposure of the blot (Fig. 5, right). 


\section{Discussion}

Fgfrl1 is a novel cell surface receptor that is structurally related to the family of the Fgfrs. Owing to this structural relationship, we and others have speculated that it might be involved in the modulation of FGF signaling $(1,3)$.

Here we demonstrate that Fgfrl1 is expressed in most tissues of late mouse embryos, but only at very low level. Nevertheless, the expression is high enough to be detectable by Northern blotting. In addition to this basal expression, cartilage and some muscle types express Fgfrll at fairly high level such that the expression becomes detectable by in situ hybridization. Permanent cartilage from the nose, the ribs and the tracheal rings as well as intermediate cartilage from the primordia of bones contain relatively high levels of the Fgfrl1 mRNA. In the case of muscle, the expression appears to vary between different muscle types. Some muscle types, including the intercostal muscles, the muscle of the tongue and the muscle of the diaphragm, express relatively high levels of Fgfrl1, whereas skeletal muscles from the limbs express it at very low levels. In fact, the musculus biceps femoris showed the lowest level of the Fgfrl1 mRNA among all the samples tested in our study. The differences in the relative expression cannot simply be explained by different developmental stages of the particular muscle types because the tongue showed particularly high expression at embryonic day 16 as well as 2 weeks after birth, whereas the muscles of the leg showed very low expression at both stages and even in adult animals (data not shown). It is therefore likely that some functional differences exist between Fgfrl1 positive and Fgfrl1 negative muscles. The tongue and to some extent also the diaphragm contain multiple interwoven muscle bundles that point in diverse directions $(13,14)$, whereas the muscles from the leg contain bundles that are aligned in parallel relative to each other. It remains to be determined whether the orientation of the bundles are linked in some way with the relative expression of Fgfrl1.

Another question relates to the common function of Fgfrl1 in two tissues as different as cartilage and muscle. Chondrocytes from cartilage are known to express primarily Fgfr3. On the other hand, myoblasts derived from skeletal muscle express primarily Fgfr1 and Fgfr4. If our hypothesis is correct that Fgfrl1 might interact with another Fgfr to modulate FGF signaling (see Introduction) we may speculate that it must be able to combine with more than one Fgfr subtype. It is therefore conceivable that the functions exerted by the novel receptor might differ depending on the particular Fgfr subtype that is expressed in cartilage and muscle.

Recently, Hayashi and coworkers (15) reported on the expression of the homologous receptor from Xenopus termed XFGFRL1. These authors detected expression in the anterior mesendodermal region at early developmental stages (gastrula, neurula). During the tail bud stage, the XFGFRL1 mRNA was found in distinct regions of the forebrain, the eyes, the midbrain-hindbrain boundary, the otic vesicles, the visceral arches and the somites. It is difficult to compare these results with our expression pattern. We did not observe any expression in the brain, the eyes or the spinal cord of late mouse embryos (15-17 days), but we found expression in cartilaginous structures. At an earlier stage (12 days), we could not detect any signal above background at all. However, mouse embryos of very early stages were not included in our study since this would have required more elaborate techniques such as in vitro fertilization. Thus, it remains to be determined whether Fgfrl1 will also play a role in the mouse during formation of the neuroectoderm and the central nervous system.

\section{Acknowledgments}

This work was supported by the Swiss National Science Foundation (3100A0-102251), the Swiss Foundation for Research on Muscular Diseases, Oncosuisse (OCS-0121102-2002) and the Novartis Foundation (04A02).

\section{References}

1. Wiedemann M and Trueb B: Characterization of a novel protein (FGFRL1) from human cartilage related to FGF receptors. Genomics 69: 275-279, 2000.

2. Trueb B, Neuhauss SCF, Baertschi S, Rieckmann T, Schild C and Taeschler S: Fish possess multiple copies of fgfrl1, the gene for a novel FGF receptor. Biochim Biophys Acta 1727: 65-74, 2005.

3. Sleeman M, Fraser J, McDonald M, Yuan S, White D, Grandison P, Kumble K, Watson JD and Murison JG: Identification of a new fibroblast growth factor receptor, FGFR5. Gene 271: 171-182, 2001.

4. Kim I, Moon S-O, Yu K-H, Kim U-H, Koh GY: A novel fibroblast growth factor receptor-5 preferentially expressed in the pancreas. Biochim Biophys Acta 1518: 152-156, 2001.

5. Szebenyi $\mathrm{G}$ and Fallon JF: Fibroblast growth factors as multifunctional signaling factors. Int Rev Cytol 185: 45-106, 1999.

6. Ornitz DM and Itoh N: Fibroblast growth factors. Genome Biol 2: 3005.1-3005.12, 2001.

7. Trueb B, Zhuang L, Taeschler S and Wiedemann M: Characterization of FGFRL1, a novel FGF receptor preferentially expressed in skeletal tissues. J Biol Chem 278: 33857-33865, 2003.

8. Wiedemann M and Trueb B: The mouse Fgfrl1 gene coding for a novel FGF receptor-like protein. Biochim Biophys Acta 1520: 247-250, 2001

9. Chomczynski P and Sacchi N: Single-step method of RNA isolation by acid guanidinium thiocyanate-phenol-chloroform extraction. Anal Biochem 162: 156-159, 1987.

10. Ausubel FM, Brent R, Kingston RE, Moore DD, Seidman JG, Smith JA and Struhl K: Current Protocols in Molecular Biology. Greene Publishing Associates, New York, 1987.

11. Feinberg AP and Vogelstein B: A technique for radiolabeling DNA restriction endonuclease fragments to high specific activity. Anal Biochem 132: 6-13, 1983.

12. Wälchli C, Koch M, Chiquet M, Odermatt BF and Trueb B: Tissue specific expression of the fibril-associated collagens XII and XIV. J Cell Sci 107: 669-681, 1994.

13. Saito $H$ and Itoh I: Three-dimensional architecture of the intrinsic tongue muscles, particularly the longitudinal muscle, by the chemical-maceration method. Anat Sci Int 78: 168-176, 2003.

14. Celli B: The diaphragm and respiratory muscles. Chest Surg Clin N Am 8: 207-224, 1998.

15. Hayashi S, Itoh M, Taira S, Agata K and Taira M: Expression patterns of Xenopus FGF receptor-like 1/nou-darake in early Xenopus development resembles those of planarian nou-darake and Xenopus FGF8. Dev Dynamics 230: 700-707, 2004.

16. Kaufman MH: The Atlas of Mouse Development. 4th edition. Academic Press, San Diego, 2001. 